\title{
On the Competitive Ratio of the Random Sampling Auction
}

\author{
Uriel Feige $^{1}$, Abraham Flaxman ${ }^{2}$, Jason D. Hartline ${ }^{3}$, and Robert Kleinberg ${ }^{4}$ \\ 1 Microsoft Research, Redmond, WA 98052. urifeige@microsoft.com \\ 2 Carnegie Mellon University, Dept. of Computer Science, Pittsburgh, PA 15213. \\ abie@cmu.edu \\ 3 Microsoft Research, Mountain View, CA, 94043. \\ hartline@microsoft.com. \\ 4 Cornell University, Dept. of Computer Science, Ithaca, NY 14853. \\ rdk@cs. cornell. edu.
}

\begin{abstract}
We give a simple analysis of the competitive ratio of the random sampling auction from [10]. The random sampling auction was first shown to be worst-case competitive in [9] (with a bound of 7600 on its competitive ratio); our analysis improves the bound to 15. In support of the conjecture that random sampling auction is in fact 4-competitive, we show that on the equal revenue input, where any sale price gives the same revenue, random sampling is exactly a factor of four from optimal.
\end{abstract}

\section{Introduction.}

Random sampling is the most prevalent technique for designing auctions to maximize the auctioneer's profit when the bidders' valuations are a priori unknown $[2-4,7,8,10,11]$. The first and simplest application of random sampling to auctions is in the context of auctioning a digital good. ${ }^{5}$ For this problem, the random sampling optimal price auction (RSOP) from [10] works by selecting a bipartition of the bidders uniformly at random and offering the optimal sale price for each part to the other part.

It is well known that, on many classes of interesting inputs, RSOP performs very close to optimally $[2,12]$. Further, it was shown in [9] that RSOP is always within a constant factor of a natural benchmark for optimality even on worstcase inputs. Their analysis is not tight; they obtain an upper bound of 7600 on the competitive ratio of RSOP, well shy of the current conjectured ratio of four.

There are a number of compelling reasons for trying to prove the conjecture that RSOP is 4-competitive. First, it is one of the most natural profit-maximizing auctions, and having a tight analysis of its performance is interesting in itself. Second, an auction that is near optimal on many natural inputs and never very bad in the worst case has practical value. Finally, because of its simplicity, RSOP, is easily adapted to other more general settings which benefit from improved

\footnotetext{
${ }^{5}$ Or any good where there are more units for sale than there are bidders.
} 
analysis of RSOP for digital goods (E.g., double auctions [3], online limited supply auctions [11], and combinatorial auctions $[2,8])$.

In this paper we give a new analysis of the random sampling optimal price auction for digital goods that tightens the upper bound on the competitive ratio to 15. Specifically, we show that the expected profit of RSOP is at least a factor of 15 of $\mathcal{F}^{(2)}$, the benchmark profit of the optimal single price sale of at least two items. We also show that on the pathological input where any single sale price gives the same revenue, i.e., the equal revenue input, the expected profit of the random sampling auction is at least $\mathcal{F}^{(2)} / 4$. We refer the reader to [9] for motivation and discussion of this analysis framework and the choice of profit benchmark.

\section{Preliminaries.}

We are considering auctioning a digital good to $n$ bidders. Since the random sampling auction is incentive compatible we assume that each bidder bids their true valuation for the good. Let $\mathbf{b}=\left(b_{1}, \ldots, b_{n}\right)$ be the vector of bids sorted in decreasing order. Consider the following definitions from the literature $[7,10]$.

Definition 1 (RSOP). The random sampling optimal price auction uniformly partitions the bidders into two parts, computes the optimal sale price for each part, and offers this sale price to the bidders in the opposite part.

Definition $2\left(\mathcal{F}^{(2)}\right)$. The profit from the optimal single price sale of at least two items is:

$$
\mathcal{F}^{(2)}(\mathbf{b})=\max _{i \geq 2} i b_{i} .
$$

Definition 3 (Competitive Ratio). The competitive ratio of an auction is the minimum value $\beta$ for which the expected profit of the auction on any input is at least $\mathcal{F}^{(2)} / \beta$. An auction is $\beta$-competitive if its competitive ratio is at most $\beta$.

Definition 4 (Equal-revenue Input). The equal revenue input is the bid vector $\mathbf{b}=\left(b_{1}, \ldots, b_{n}\right)$ with $b_{i}=1 / i$.

We will be employing this competitive framework to analyze the performance of RSOP. Our main result is the proof of the following theorem.

Theorem 1. RSOP is 15-competitive.

In proving Theorem 1 and analyzing RSOP on the equal revenue input, we will use an analytical tool which gives an exact computation of the probability of an event, $\mathcal{E}_{\alpha}$, defined as follows. Consider a discrete random walk on a line such that in each time step, the walk takes one step forward or stays put, independently with probability $1 / 2$. If we start at the origin at time $i=1$, then $\mathcal{E}_{\alpha}$ is the event that at no time $i \geq 1$ is the random walk further than $\alpha i$ from the origin. We prove the following lemma. 


\section{Lemma 1.}

$$
\begin{aligned}
\operatorname{Pr}\left[\mathcal{E}_{\frac{3}{4}}\right] & =1-\frac{1}{81}\left((17+3 \sqrt{33})^{1 / 3}-1-2(17+3 \sqrt{33})^{-1 / 3}\right)^{4} \\
& =0.912622 \pm 4 \times 10^{-6} .
\end{aligned}
$$

It seems likely that this lemma was known prior to the present work, though we are not aware of any proof which has previously appeared elsewhere. Our proof technique provides a closed-form value of $\operatorname{Pr}\left[\mathcal{E}_{\alpha}\right]$ for a limited range of $\alpha$ values. For $\alpha$ of the form $\frac{k-1}{k}$ we find the value of $\operatorname{Pr}\left[\mathcal{E}_{\alpha}\right]$ implicitly as the unique root of a $k$-th degree polynomial on the interval $(0,1)$. For arbitrary values of $\alpha$ we describe a computer aided proof that $\operatorname{Pr}\left[\mathcal{E}_{\alpha}\right]$ lies in an interval of width $\epsilon$ (with proof length proportional to $\log (1 / \epsilon)$ ).

\section{Proof of Lemma 1.}

Let $S_{i}$ be the variable for the position of a random walk on a line that starts at the origin at time $i=1$ and proceeds in each round to stay put or move forward each with probability $1 / 2$. The lemma calls for the calculation of the probability, $\operatorname{Pr}\left[\mathcal{E}_{\alpha}\right]$, that for all $i, S_{i} / i \leq \alpha$, in particular for $\alpha=\frac{3}{4}$.

For any $\alpha$ that takes the form $\alpha=\frac{k-1}{k}$ where $k$ is an integer, we can rewrite $\mathcal{E}_{\alpha}$ as the event that for all $i,(k-1)\left(i-S_{i}\right)-S_{i} \geq 0$. By setting $Z_{i}=(k-$ 1) $\left(i-S_{i}\right)-S_{i}$, we have that $\left(Z_{1}, Z_{2}, Z_{3}, \ldots\right)$ is a random walk which increases by $k-1$ with probability $1 / 2$ and otherwise decreases by 1 (where, because $S_{1}=0$, the walk starts with $Z_{1}=k-1$ ). We also note that $\operatorname{Pr}\left[\overline{\mathcal{E}}_{\alpha}\right]$ is equal to the probability of ruin for this asymmetric random walk, which we denote by $p_{k}$ (for a general study of the probability of ruin, see for example [6, Chapter $\mathrm{XIV])}$. In general, let

$$
p_{j}=\operatorname{Pr}\left[\text { exists } i, Z_{i}=Z_{1}-j\right] .
$$

Because the random walk is memoryless and never decreases by more than 1 , $p_{j}=\left(p_{1}\right)^{j}$ for $j>0$. By expanding the probability conditionally on the value of $S_{2}$, we have

$$
\begin{aligned}
p_{1} & =\frac{1}{2}+\frac{1}{2} p_{k} \\
& =\frac{1}{2}\left(1+p_{1}^{k}\right) .
\end{aligned}
$$

The polynomial $f(x)=x^{k}-2 x+1$ has $f(0)=1$ and $f(1)=0$. Since $f^{\prime}(x)=$ $k x^{k-1}-2$ has one root on the interval $(0,1)$ and $f^{\prime \prime}(x)=k(k-1) x^{k-2}$ is positive on this interval, there is a unique root of $f$ on the interval $(0,1)$; call this root $r$. Since $0 \leq p_{1} \leq 1$ and $f\left(p_{1}\right)=0$, we must have either $p_{1}=1$ or $p_{1}=r$. We will prove that $p_{1}<1$, thus establishing that $p_{1}=r$. Let $\left(Y_{1}, Y_{2}, \ldots\right)$ be the absorbing random walk given by $Y_{i}=Z_{i}-k+2$ until it drops to $Y_{i}=0$, where it stays. More formally,

$$
Y_{i}= \begin{cases}0, & \text { if } Y_{j}=0 \text { for some } j<i \\ Z_{i}-Z_{1}+1, & \text { otherwise. }\end{cases}
$$


Let $W_{i}$ be the random variable given by $W_{i}=r^{Y_{i}}$. We claim $\left(W_{1}, W_{2}, \ldots\right)$ satisfies the martingale property that $\mathrm{E}\left[W_{i+1} \mid W_{i}\right]=W_{i}$. This holds trivially when $Y_{i}=0$. For $Y_{i}>0$, the equation $\mathrm{E}\left[W_{i+1} \mid W_{i}\right]=W_{i}$ follows from the calculation

$$
\mathrm{E}\left[r^{Z_{i+1}} \mid Z_{i}\right]=\frac{1}{2} r^{Z_{i}-1}+\frac{1}{2} r^{Z_{i}+k-1}=\frac{1}{2}\left(r^{-1}+r^{k-1}\right) r^{Z_{i}}=r^{Z_{i}} .
$$

(The final equality follows from $f(r)=r^{k}-2 r+1=0$.) Since $\left(W_{1}, W_{2}, \ldots\right)$ is a martingale we have $\mathrm{E}\left[W_{t}\right]=W_{1}=r$ for all $t \geq 1$.

Now let

$$
p_{1, t}=\operatorname{Pr}\left[\text { exists } i \leq t, Z_{i}=Z_{1}-1\right]=\operatorname{Pr}\left[W_{t}=1\right] .
$$

Defining $\mathcal{A}_{t}$ to be the event that $Z_{t}=Z_{1}-1$ and $Z_{s}>Z_{t}$ for all $s<t$, we see that the events $\mathcal{A}_{t}$ are disjoint and measurable, and that $p_{1, t}=\sum_{i=1}^{t} \operatorname{Pr}\left(\mathcal{A}_{i}\right)$ and $p_{1}=\sum_{i=1}^{\infty} \operatorname{Pr}\left(\mathcal{A}_{i}\right)$. This establishes that $p_{1}=\lim _{t \rightarrow \infty} p_{1, t}$ while also confirming that $\mathcal{E}_{\alpha}$ is a measurable event since it is the union of the events $\mathcal{A}_{i}(1 \leq i<\infty)$.

Since $W_{t}$ is a non-negative random variable, we have $p_{1, t}=\operatorname{Pr}\left[W_{t}=1\right] \leq$ $\mathrm{E}\left[W_{t}\right]=r$. Recalling that $p_{1}=\lim _{t \rightarrow \infty} p_{1, t}$, we conclude that $p_{1} \leq r<1$, as claimed. This completes the proof that $p_{1}=r$ and that $p_{j}=r^{j}$ for all $j \geq 1$.

Recall that to calculate $\operatorname{Pr}\left[\mathcal{E}_{\frac{3}{4}}\right]$ we are intersted in the case that $k=4$. When $k=4$ the polynomial $f$ is a quartic equation, and the root $r$ is given exactly by Ferrari's formula [5]. So

$$
p_{1}=\frac{1}{3}\left[(17+3 \sqrt{33})^{1 / 3}-1-2(17+3 \sqrt{33})^{-1 / 3}\right]
$$

and to complete the proof we have

$$
\begin{aligned}
\operatorname{Pr}\left[\mathcal{E}_{\frac{3}{4}}\right]=1-p_{4} & =1-p_{1}^{4} \\
& =1-\frac{1}{81}\left[(17+3 \sqrt{33})^{1 / 3}-1-2(17+3 \sqrt{33})^{-1 / 3}\right]^{4} .
\end{aligned}
$$

\section{Proof of Theorem 1.}

In our random partition, we call the side of the bipartition with $b_{1}$ on it the bad side, and we call the other side the good side. We may assume that $b_{1}$ is larger than $\mathcal{F}^{(2)}$, since this can only increase the gap between the expected profit of RSOP and $\mathcal{F}^{(2)}$. (Increasing $b_{1}$ can not change $\mathcal{F}^{(2)}$, nor can it change the revenue obtained by RSOP from bidders on the bad side of the bipartition; moreover, if $b_{1}$ is sufficiently large then RSOP will obtain zero revenue from bidders on the good side of the bipartition.) Let $X_{i} \in\{0,1\}$ be an indicator random variable for the event that bidder $i$ is on the good side. By definition $X_{1}=0$. Let $S_{i}=\sum_{j=1}^{i} X_{j}$ be the number of bidders with bid at least $b_{i}$ on the good side. Note that $S_{i}$ is a random variable that behaves like the random walk under consideration in Lemma 1 . Let $i^{\star}$ denote the index of the bidder whose 
value $b_{i^{\star}}$ maximizes revenue on the good side (meaning $S_{i^{\star}} b_{i^{\star}} \geq S_{j} b_{j}$ for all $j$, with ties broken arbitrarily).

Then the profit of the random sampling auction is

$$
R S=\left(i^{\star}-S_{i^{\star}}\right) b_{i^{\star}} .
$$

We now show that $\mathbf{E}[R S] \geq \mathcal{F}^{(2)} / 15$.

Let $i^{\prime}$ denote the index of the bidder whose value $b_{i^{\prime}}$ is the optimal sale price for the full set of bids (meaning $i^{\prime} b_{i^{\prime}} \geq j b_{j}$ for all $j \geq 2$, with ties broken arbitrarily). We shall first provide a bound for the case when $i^{\prime}$ is even, and later explain how the same bound (or in fact, a slightly better one) can be obtained when $i^{\prime}$ is odd.

Consider the event $\mathcal{B}=\left\{S_{i^{\prime}} \geq i^{\prime} / 2\right\}$. Using the fact that $i^{\prime}$ is even, it follows that $\operatorname{Pr}[\mathcal{B}]=1 / 2$ (because the event holds when the majority of $i^{\prime}$ highest bidders other than the largest bid are on the good side). In this case, the optimal single price profit from the good side is

$$
S_{i^{\star}} b_{i^{\star}} \geq S_{i^{\prime}} b_{i^{\prime}} \geq \mathcal{F}^{(2)} / 2 .
$$

To avoid unnecessary subscripts, we set $\mathcal{E}=\mathcal{E}_{\frac{3}{4}} .{ }^{6}$ If event $\mathcal{E}$ occurs, then, for all $i$, we have

$$
\left(i-S_{i}\right) b_{i} \geq \frac{1}{4} i b_{i} \geq \frac{1}{3} S_{i} b_{i} .
$$

If both $\mathcal{E}$ and $\mathcal{B}$ occur then

$$
R S=\left(i^{\star}-S_{i^{\star}}\right) b_{i^{\star}} \geq \frac{1}{3} S_{i^{\star}} b_{i^{\star}} \geq \mathcal{F}^{(2)} / 6 .
$$

Thus, the expected profit of RSOP is at least

$$
\mathrm{E}[R S]=\mathrm{E}\left[\left(i^{\star}-S_{i^{\star}}\right) b_{i^{\star}}\right] \geq \operatorname{Pr}[\mathcal{E} \cap \mathcal{B}] \mathcal{F}^{(2)} / 6 .
$$

By Lemma $1, \operatorname{Pr}[\mathcal{E}] \geq 0.9$, so

$$
\operatorname{Pr}[\mathcal{E} \cap \mathcal{B}]=1-\operatorname{Pr}[\overline{\mathcal{E}} \cup \overline{\mathcal{B}}] \geq 1-\operatorname{Pr}[\overline{\mathcal{E}}]-\operatorname{Pr}[\overline{\mathcal{B}}]=\operatorname{Pr}[\mathcal{E}]-\frac{1}{2} \geq 0.4 .
$$

Therefore $\mathrm{E}[R S] \geq \mathcal{F}^{(2)} / 15$.

We now address the case when $i^{\prime}$ is odd. In this case we consider the event $\mathcal{C}=\left\{S_{i^{\prime}} \geq\left(i^{\prime}-1\right) / 2\right\}$. It is not hard to see that $\operatorname{Pr}[\mathcal{C}]=1 / 2+2^{-i^{\prime}}\left(\begin{array}{c}i^{\prime}-1 \\ \left(i^{\prime}-1\right) / 2\end{array}\right)$. It can be verified that for every odd $i^{\prime} \geq 3$, a straightforward modification of the proof that was given for the case that $i^{\prime}$ is even gives a bound that is at least $1 / 15$. This completes the proof of the theorem.

\footnotetext{
${ }^{6}$ It is possible to perform this calculation with the event $\mathcal{E}_{\alpha}$ for $\alpha \neq \frac{3}{4}$. This produces an upper bound on the competitive ratio of RSOP of $\left[\left(\operatorname{Pr}\left[\mathcal{E}_{\alpha}\right]-\frac{1}{2}\right)\left(\frac{1-\alpha}{\alpha}\right)\right]^{-1}$. Computer calculations following the same flavor as those made in Section 5 suggest this is minimized when $\alpha=\frac{3}{4}$.
} 


\section{Random Sampling and the Equal Revenue Input.}

We now discuss the performance of RSOP on the equal revenue input (See Definition 4). This input is of particular interest because the intuition motivating random sampling does not apply to it. A price that looks good for one part, because disproportionately many bids are above it, is a bad price for the other part because disproportionately few bids are above it. In this section we discuss a computer-aided calculation that shows that the RSOP on the equal revenue input has expected profit at least $\mathcal{F}^{(2)} / 4$ (and this is tight when there are only $n=2$ bidders). Note that on the equal revenue input, $n$ bidders with $b_{i}=1 / i$, the optimal single price sale obtains profit $\mathcal{F}^{(2)}(\mathbf{b})=1$.

Define the event $\mathcal{E}_{\alpha}^{n}=\left\{\right.$ for all $\left.i \leq n, \frac{S_{i}}{i} \leq \alpha\right\}$ with $S_{i}$ as defined in previous sections. Fix a positive integer $N$ and let $\alpha_{i}=i / N$. Then let $\mathcal{A}_{i}^{n}=\mathcal{E}_{\alpha_{i}}^{n} \cap \overline{\mathcal{E}}_{\alpha_{i-1}}^{n}$ be the event that some $S_{i} / i$ exceeds $\alpha_{i-1}$ but none exceed $\alpha_{i}$. Thus, $\operatorname{Pr}\left[\mathcal{A}_{i}^{n}\right]=$ $\operatorname{Pr}\left[\mathcal{E}_{\alpha_{i}}^{n}\right]-\operatorname{Pr}\left[\mathcal{E}_{\alpha_{i-1}}^{n}\right]$. The events $\mathcal{A}_{i}^{n}$ are disjoint and if $\mathcal{A}_{i}^{n}$ holds then $\left(1-\alpha_{i}\right) \leq$ $R S \leq\left(1-\alpha_{i-1}\right)$. Therefore, the profit of RSOP on the $n$ bid equal revenue input satisfies

$$
\sum_{i=1}^{N-1} \operatorname{Pr}\left[\mathcal{A}_{i}^{n}\right]\left(1-\alpha_{i}\right) \leq \mathrm{E}[R S] \leq \sum_{i=1}^{N-1} \operatorname{Pr}\left[\mathcal{A}_{i}^{n}\right]\left(1-\alpha_{i-1}\right)
$$

What remains is to show how we can calculate $\operatorname{Pr}\left[\mathcal{E}_{\alpha}^{n}\right]$ and extend the above discussion to get a bound $\mathrm{E}[R S]$ for the equal revenue input with any $n$.

\section{Calculating $\operatorname{Pr}\left[\mathcal{E}_{\alpha}^{n}\right]$ for general $\alpha$.}

The proof in Section 3 can be easily adapted to give an implicit value of $\operatorname{Pr}\left[\mathcal{E}_{\alpha}\right]$ whenever $\alpha=\frac{k-1}{k}$ for some integer $k$. When $k \leq 5$, it is possible to turn this into a closed-form solution. We now describe an alternative method for calculating $\operatorname{Pr}\left[\mathcal{E}_{\alpha}^{n}\right]$ which leads to bounds on $\operatorname{Pr}\left[\mathcal{E}_{\alpha}\right]$ that do not require $\alpha$ to be of any special form,

Fix a value of $\alpha$. To bound the value of $\operatorname{Pr}\left[\mathcal{E}_{\alpha}^{n}\right]$, we define

$$
p(i, j)=\operatorname{Pr}\left[S_{i}=j \cap S_{i^{\prime}} \leq \alpha i^{\prime} \text { for all } i^{\prime} \leq i\right]
$$

and note that $\operatorname{Pr}\left[\mathcal{E}_{\alpha}^{n}\right]=\sum_{j=0}^{n} p(n, j)$. We now can use a standard computer algebra package, like Mathematica, to evaluate $\operatorname{Pr}\left[\mathcal{E}_{\alpha}^{n}\right]$ using the following recurrence. The initial conditions are derived from the fact our random walk starts at time $i=1$ (i.e., $S_{1}=0$ ).

$$
\begin{aligned}
& p(i, j)= \begin{cases}\frac{1}{2} p(i-1, j-1)+\frac{1}{2} p(i-1, j), & \text { if } 0 \leq j \leq \alpha i \\
0, & \text { otherwise }\end{cases} \\
& p(1, j)= \begin{cases}1, & j=0 ; \\
0, & \text { otherwise. }\end{cases}
\end{aligned}
$$


For reasonable values of $n$, it is possible to evaluate $\operatorname{Pr}\left[\mathcal{E}_{\alpha}^{n}\right]$. For example, for $\alpha=\frac{3}{4}, \operatorname{Pr}\left[\mathcal{E}_{\frac{3}{4}}^{200}\right]$ equals

$\frac{22914483922452727752710576603653551719219315819721902777499}{25108406941546723055343157692830665664409421777856138051584}$.

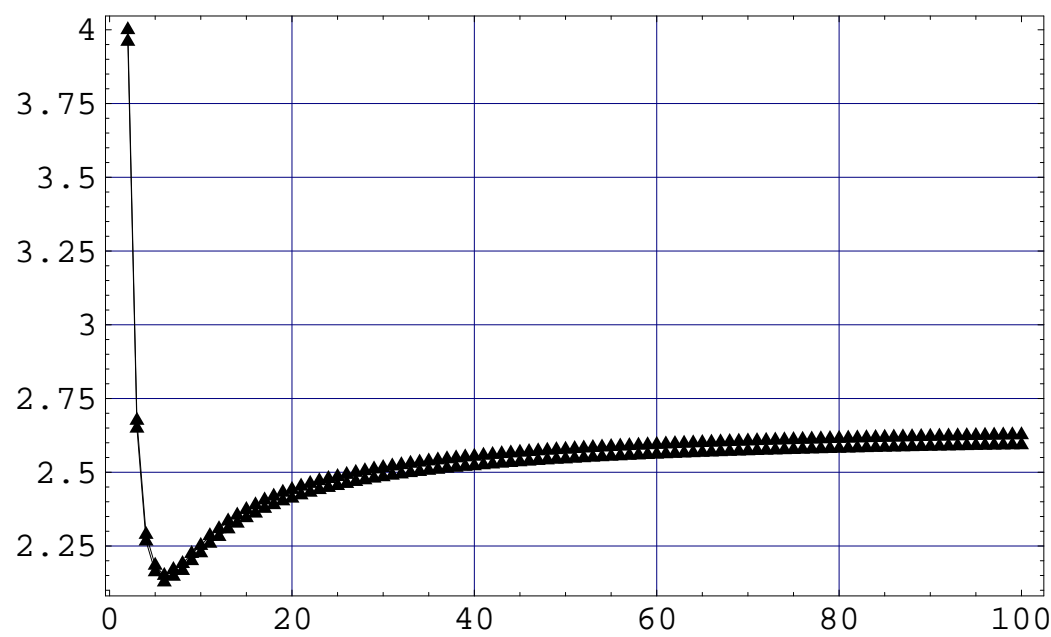

Fig. 1. Upper and lower bounds on $\mathcal{F}^{(2)} / \mathrm{E}[R S]$ when $N=200$ for equal revenue input with $n=2, \ldots, 100$.

\section{Bounding $\mathrm{E}[\boldsymbol{R S}]$ for all $n$.}

Using the recurrence relationship for $\operatorname{Pr}\left[\mathcal{E}_{\alpha}^{n}\right]$ with different values of $\alpha$ and equation (1), we can calculate $\operatorname{Pr}\left[\mathcal{A}_{i}^{n}\right]$ and $\mathrm{E}[R S]$ for any given $n$. This calculation shows that as $n$ increases, the ratio $\mathcal{F}^{(2)} / \mathrm{E}[R S]$ is not monotonic. A plot of the upper and lower bounds on $\mathcal{F}^{(2)} / \mathrm{E}[R S]$ obtained by taking $N=200$ appears in Figure 1. For $N=200$ and $n=100$ this calculation shows that $2.59 \leq \mathcal{F}^{(2)} / \mathrm{E}[R S] \leq 2.63$ and growing. To extend this bound to all values of $n$, we must still prove that for large $n$ the ratio does not eventually grow bigger than four.

To do so, we get bounds on $\operatorname{Pr}\left[\mathcal{E}_{\alpha}\right]$ in terms of $\operatorname{Pr}\left[\mathcal{E}_{\alpha}^{n}\right] . \operatorname{Pr}\left[\mathcal{E}_{\alpha}^{n}\right]$ is an upper bound of $\operatorname{Pr}\left[\mathcal{E}_{\alpha}\right]$. We can get a lower bound by applying the union bound as 
follows:

$$
\begin{aligned}
1-\operatorname{Pr}\left[\mathcal{E}_{\alpha}^{n}\right] \leq \operatorname{Pr}\left[\overline{\mathcal{E}}_{\alpha}\right] & \leq 1-\operatorname{Pr}\left[\mathcal{E}_{\alpha}^{n}\right]+\sum_{i \geq n} \operatorname{Pr}\left[S_{i} \geq \alpha i\right] \\
& \leq 1-\operatorname{Pr}\left[\mathcal{E}_{\alpha}^{n}\right]+\sum_{i \geq n} e^{-(\alpha-1 / 2)^{2} i / 3} \\
& =1-\operatorname{Pr}\left[\mathcal{E}_{\alpha}^{n}\right]+\frac{e^{-(\alpha-1 / 2)^{2} n / 3}}{1-e^{-(\alpha-1 / 2)^{2} / 3}}
\end{aligned}
$$

As an aside, an alternate proof of Lemma 1 can be obtained from the above bound with $n=800$ to show that

$$
\operatorname{Pr}\left[\mathcal{E}_{\frac{3}{4}}\right]=0.912622 \pm 4 \times 10^{-6}
$$

We now use these upper and lower bounds on $\operatorname{Pr}\left[\mathcal{E}_{\alpha}\right]$ to get a lower bound on $\operatorname{Pr}\left[\mathcal{A}_{\alpha}\right]$. For any $n \geq n_{0}$,

$$
\operatorname{Pr}\left[\mathcal{A}_{i}^{n}\right] \geq \operatorname{Pr}\left[\mathcal{E}_{\alpha_{i}}\right]-\operatorname{Pr}\left[\mathcal{E}_{\alpha_{i-1}}^{n_{0}}\right] \geq \operatorname{Pr}\left[\mathcal{E}_{\alpha_{i}}^{n_{0}}\right]-\frac{e^{-\left(\alpha_{i}-1 / 2\right)^{2} n_{0} / 3}}{1-e^{-\left(\alpha_{i}-1 / 2\right)^{2} / 3}}-\operatorname{Pr}\left[\mathcal{E}_{a_{i-1}}^{n_{0}}\right]
$$

This bound is only useful when $\alpha_{i}>1 / 2$ and $n_{0}$ is sufficiently large. Fortunately, to get a good lower bound on $\mathrm{E}[R S]$ for large $n$ it suffices to consider only the contribution to the sum in equation (1) from the terms with high $\alpha$. For sufficiently large $i_{0}$ and $n_{0}$ we are left with the bound:

$$
\mathrm{E}[R S] \geq\left(1-\alpha_{i_{0}}\right) \operatorname{Pr}\left[\mathcal{E}_{\alpha_{i_{0}}}\right]+\sum_{i=i_{0}+1}^{N-1} \operatorname{Pr}\left[\mathcal{A}_{i}^{n_{0}}\right]\left(1-\alpha_{i}\right)
$$

Taking $n_{0}=500, N=100$, and $i_{0}=70$ (so $\left.\alpha_{i_{0}}=0.7\right)$ and using the computer to prove bounds on the terms in this sum shows that for all $n \geq 500$, $\mathrm{E}[R S] \geq \mathcal{F}^{(2)} / 3.6$. This, combined with the computer proof outlined previously for $n \leq n_{0}$, completes the proof showing that RSOP is 4-competitive on the equal revenue input.

\section{Conclusions}

As we mentioned in the introduction, the random sampling technique is widely applicable to the design of profit maximizing mechanisms. The basic RSOP auction has been generalized and applied to the problem of designing double auctions [3], online limited supply auctions [11], multi-unit auctions for bidders with budget constraints [4], combinatorial auctions [2,8], and knapsack auctions [1]. With exception of the work of Hajiaghayi et al. [11] on online limited supply auctions, all of these generalized applications of RSOP are given with analyses that obtain promise style bounds. A typical promise style bound would state that if $n$, the number of bidders, is large enough then the random sampling 
auction's profit is near optimal. No bound is given if the promise is not met. There are several reasons for using such a promise bound. First, it allows for $(1-\epsilon)$-approximations, with $\epsilon$ a parameter that improves with the restrictiveness of the promise. Such bounds are of interest as $(1-\epsilon)$-approximations are not possible in the worst case competitive framework of this and preceding papers [7]. Second, the analysis of RSOP in [9] is complicated and gives such a loose bound that generalizing it to other contexts seems to be of marginal worth. In contrast, our improved analysis opens up the possibility of doing a worst case analysis of the random sampling auction for some of these more general applications.

\section{Acknowledgments}

We would like to thank Andrew Goldberg for many helpful discussions.

\section{References}

1. G. Aggarwal and J. Hartline. Knapsack Auctions. In Proc. 17th Symp. on Discrete Alg. ACM/SIAM, 2006.

2. M.-F. Balcan, A. Blum, J. Hartline, and Y. Mansour. Mechanism Design via Machine Learning. In Proc. of the 46th IEEE Symp. on Foundations of Computer Science, 2005.

3. S. Baliga and R. Vohra. Market research and market design. Advances in Theoretical Economics, 3, 2003.

4. C. Borgs, J. Chayes, N. Immorlica, M. Mahdian, and A. Saberi. Multi-unit Auctions with Budget-constrained Bidders. In Proc. of 6th ACM Conference on Electronic Commerce. ACM Press, New York, 2005.

5. G. Cardano. Ars Magna (Artis magnae sive de regulis algebrae liber unus). Nuremberg, 1545 .

6. W. Feller. An Introduction to Probability Theory and its Applications, volume 1. John Wiley and Sons, Inc., New York, 3rd edition, 1968.

7. A. Fiat, A. Goldberg, J. Hartline, and A. Karlin. Competitive Generalized Auctions. In Proc. 34th ACM Symposium on the Theory of Computing. ACM Press, New York, 2002.

8. A. Goldberg and J. Hartline. Competitive auctions for multiple digital goods. In ESA, 2001.

9. A. Goldberg, J. Hartline, A. Karlin, M. Saks, and A. Wright. Competitive auctions and digital goods. Games and Economic Behavior, 2002. To appear. An earlier version appeared as InterTrust Technical Report STAR-TR-99-09-01.

10. A. Goldberg, J. Hartline, and A. Wright. Competitive Auctions and Digital Goods. In Proc. 12th Symp. on Discrete Alg., pages 735-744. ACM/SIAM, 2001.

11. M. Hajiaghayi, R. Kleinberg, and D. Parkes. Adaptive Limited-Supply Online Auctions. In Proc. of 5th ACM Conference on Electronic Commerce. ACM Press, New York, 2004.

12. I. Segal. Optimal pricing mechanisms with unknown demand. American Economic Review, 16:50929, 2003. 